University of Nebraska - Lincoln

DigitalCommons@University of Nebraska - Lincoln

Faculty Publications: Materials Research

Science and Engineering Center

Materials Research Science and Engineering

Center

$10-27-2003$

\title{
Spin blockade in ferromagnetic nanocontacts
}

\author{
M. Ye Zhuralev \\ University of Nebraska - Lincoln \\ Evgeny Y. Tsymbal \\ University of Nebraska-Lincoln, tsymbal@unl.edu \\ Sitaram Jaswal \\ University of Nebraska, sjaswal1@unl.edu
}

A.V. Vedyayev
CEA/Grenoble, Departement de Recherche Fondamentale sur la Matiere Condensee, SPINTEC
B. Dieny
CEA/Grenoble, Departement de Recherche Fondamentale sur la Matiere Condensee, SPINTEC

Follow this and additional works at: https://digitalcommons.unl.edu/mrsecfacpubs

Part of the Materials Science and Engineering Commons

Zhuralev, M. Ye; Tsymbal, Evgeny Y.; Jaswal, Sitaram; Vedyayev, A.V.; and Dieny, B., "Spin blockade in ferromagnetic nanocontacts" (2003). Faculty Publications: Materials Research Science and Engineering Center. 33.

https://digitalcommons.unl.edu/mrsecfacpubs/33

This Article is brought to you for free and open access by the Materials Research Science and Engineering Center at DigitalCommons@University of Nebraska - Lincoln. It has been accepted for inclusion in Faculty Publications: Materials Research Science and Engineering Center by an authorized administrator of DigitalCommons@University of Nebraska - Lincoln. 


\title{
Spin blockade in ferromagnetic nanocontacts
}

\author{
M. Ye. Zhuravlev, ${ }^{\text {a) }}$ E. Y. Tsymbal, and S. S. Jaswal \\ Department of Physics and Astronomy and Center for Materials Research and Analysis, \\ University of Nebraska, Lincoln, Nebraska 68588
}

A. V. Vedyayev ${ }^{\text {b) }}$ and B. Dieny

CEA/Grenoble, Département de Recherche Fondamentale sur la Matière Condensée, SPINTEC, 38054 Grenoble, France

(Received 10 July 2003; accepted 5 September 2003)

\begin{abstract}
Using a free-electron model and a linear response theory we investigate spin-dependent electronic transport in magnetic nanocontacts in the ballistic regime of conduction. We emphasize the fact that in atomic-size ferromagnetic contacts it is possible to achieve the conductance value of $e^{2} / h$, which implies a fully spin-polarized electric current. We explore some consequences of this phenomenon. In particular, we show that the presence of a nonmagnetic region in the nanocontact separating two ferromagnetic electrodes can lead to a spin blockade resulting in very large values of magnetoresistance. (C) 2003 American Institute of Physics. [DOI: 10.1063/1.1622986]
\end{abstract}

The electrical conductance through a narrow constriction is quantized when the constriction width is comparable to the electron Fermi wavelength. This phenomenon was discovered in two-dimensional electron gas semiconductor structures, in which the constriction width can be controlled by the gate voltage. ${ }^{1}$ The quantized conductance was also observed in metallic nanowires, where an atomic-size constriction is created by pulling off two electrodes in contact. ${ }^{2-4}$ The conductance quantization can be explained within the Landauer formula ${ }^{5}$ and the adiabatic principle ${ }^{6}$ according to which the conductance is given by $\Gamma=N e^{2} / h$, where $N$ is the number of open conducting channels, i.e., the number of transverse modes at the Fermi energy. When the constriction width changes the number of conducting channels and consequently the conductance vary in discrete steps. For diamagnetic nanowires the conductance is quantized in units of $2 e^{2} / h$, where the factor 2 stands for spin degeneracy. If the constriction is made of a ferromagnetic metal, such as $\mathrm{Ni}$, the exchange energy lifts the spin degeneracy and the conductance is quantized in units of $e^{2} / h$. Such a phenomenon was observed in Ni break junctions, ${ }^{7} \mathrm{Ni}$ nanowires electrodeposited into pores of membranes, ${ }^{8} \mathrm{Ni}$ atomic-size contacts made by a scanning tunneling microscope, ${ }^{9}$ and electrodeposited $\mathrm{Ni}$ nanocontacts grown by filling an opening in focused-ionbeam-milled nanowires. ${ }^{10}$

An interesting observation that follows from these studies is the possibility to achieve the conductance value of $e^{2} / h$, which implies that one spin channel is open, whereas the other spin channel is closed. In this regime the ferromagnetic atomic-size constrictions resemble half-metallic bulk ferromagnets, materials for which only one spin band is occupied at the Fermi level, resulting in a perfect $100 \%$ spin polarization. ${ }^{11} \mathrm{We}$ see, therefore, that by making a sufficiently narrow nanoconstriction from an ordinary ferromagnet it is possible to achieve a fully spin-polarized electric current.

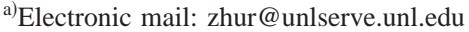

b) Permanent address: Department of Physics, M. V. Lomonosov Moscow State University, 119899 Moscow, Russia.
}

In this letter, using a free-electron model and quantummechanical description of electronic transport in the ballistic regime we explore some consequences of this phenomenon. In particular, we show that the presence of a nonmagnetic region in the nanocontact separating two ferromagnetic electrodes can lead to a spin blockade resulting in very large values of magnetoresistance.

We model nanoconstrictions by considering a segmented cylindrical nanowire of a variable radius as shown in Fig. 1(a). The electronic structure of the nanowire is described by free electrons moving in a constant potential $V_{j}$ within each

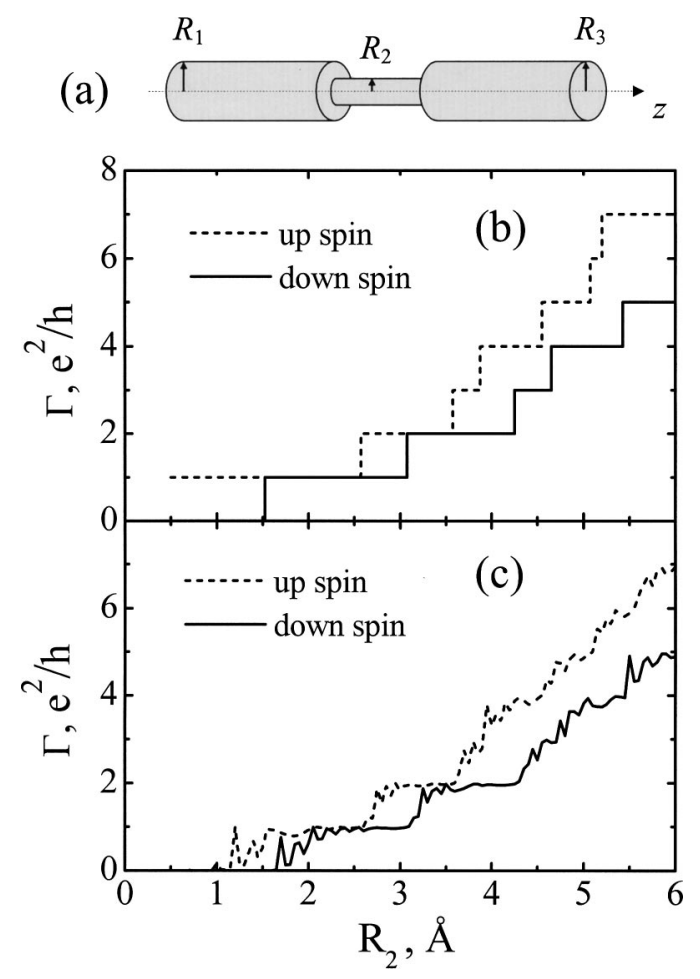

FIG. 1. (a) Segmented nanowire representing a nanoconstriction; (b) conductance for up- and down-spin electrons in a nanowire of constant radius $R=R_{1}=R_{2}=R_{3}$ as a function of $R$; (c) conductance for up- and down-spin electrons in a segmented nanowire of $R_{1}=R_{3}=15 \AA$ and the length of the middle segment $L_{2}=20 \AA$ as a function of $R_{2}$. 
segment, where index $j$ denotes the segment number. The exchange splitting of the bands in a ferromagnet is introduced using the Stoner model via an effective exchange field, $h_{e x}$, so that the spin-dependent potential within segment $j$ is $V_{\sigma j}=V_{j}+\sigma h_{e x}$, where $\sigma$ is the spin index. For an infinite wire of potential $V_{\sigma j}$ the Fermi wave vector, $k_{F}^{\sigma j}$ $=\sqrt{2 m\left(E_{F}-V_{\sigma j}\right) / \hbar^{2}}$, depends on the radius of the nanowire, $R_{j}$, through the Fermi energy $E_{F}$ which is determined by the number of valence electrons in a metal. In segmented nanowires we fix the Fermi energy throughout the structure and renormalize potentials $V_{\sigma j}$ (and consequently the Fermi momenta) to provide charge neutrality on average within each segment. We assume that there is no variation in the exchange energy with the radius of the nanowire.

We analyze the electrical conductance using a linear response theory. The central quantity to be calculated within this approach is the one-electron Green's function. Following the method developed in Ref. 12, we obtain the Green's function $G\left(r, r^{\prime}, z, z^{\prime}, \theta, \theta^{\prime}\right)$ by taking into account cylindrical symmetry of the problem. For a given segment $j$ the solution can be expanded in terms of radial and angular eigenfunctions:

$$
\begin{aligned}
& G\left(r, r^{\prime}, z, z^{\prime}, \theta, \theta^{\prime}\right) \\
& =\sum_{l m n} \frac{e^{i l\left(\theta-\theta^{\prime}\right)}}{2 \pi}\left\{\frac{\phi_{\operatorname{lm}}(r) \phi_{\operatorname{lm}}\left(r^{\prime}\right) e^{i k_{\operatorname{lm}}^{\sigma j}\left|z-z^{\prime}\right|}}{2 i k_{\operatorname{lm}}^{\sigma j}}\right. \\
& \left.\quad \times \delta_{m n}+A_{\operatorname{lm} n} \phi_{\operatorname{lm}}(r) \phi_{\ln }\left(r^{\prime}\right) e^{-i k_{\operatorname{lm}}^{\sigma j} z} e^{-i k_{\ln }^{\sigma j} z^{\prime}}\right\},
\end{aligned}
$$

where $k_{\operatorname{lm}}^{\sigma j}=\sqrt{\left(k_{F}^{\sigma j}\right)^{2}-\left(\nu_{\mathrm{lm}} / R_{j}\right)^{2}}$ is the longitudinal component of the wave vector at the Fermi energy. Radial functions $\phi_{\operatorname{lm}}(r)$ are given by

$$
\phi_{\operatorname{lm}}(r)=\sqrt{2} J_{l}\left(\frac{\nu_{1 \mathrm{~m}}}{R_{j}} r\right) / R_{j} J_{l+1}\left(\nu_{\mathrm{lm}}\right),
$$

where $\nu_{1 \mathrm{~m}}$ is the $m$ th node of the Bessel function $J_{l}(x)$. The coefficients $A_{\operatorname{lm} n}$ are calculated from the continuity condition of the Green's function and its derivatives at the interfaces. Mathematically the problem is reduced to the inversion of infinite rank matrices which can be performed using an appropriate truncation procedure. ${ }^{13}$ A similar problem was encountered when considering the conductance of $2 \mathrm{D}$ and $3 \mathrm{D}$ nanowires of variable radius using the scattering matrix formalism. ${ }^{14}$

Using the Green's function (1) we calculate spin conductance $\Gamma_{\sigma}$ at zero temperature from the Kubo formula. The result can be represented as follows:

$$
\begin{aligned}
\Gamma_{\sigma}= & -\frac{2 e^{2}}{h} \sum_{l m n}\left[\operatorname{Im}\left(k_{\operatorname{lm}}^{\sigma j}\right)\left(\left|A_{\operatorname{lm} n}\right|^{2}-\frac{\delta_{m n}}{4\left|k_{\operatorname{lm}}^{\sigma j}\right|^{2}}\right)\right. \\
& \left.+\operatorname{Re}\left(k_{\operatorname{lm}}^{\sigma j}\right) \operatorname{Im}\left(\frac{A_{\operatorname{lm} n}}{\left(k_{\operatorname{lm}}^{\sigma j}\right)^{*}}\right)\right] \operatorname{Im}\left(k_{\ln }^{\sigma j}\right) .
\end{aligned}
$$

Note that due to the current continuity condition $\Gamma_{\sigma}$ does not depend on the choice of segment $j$ in which it is calculated.

Figure 1(b) shows the calculated spin-dependent conductance of a uniformly magnetized wire of constant radius $R$ $=R_{1}=R_{2}=R_{3}$. In the calculations we used the commonly accepted values of the material parameters typical for $\mathrm{Ni}$ and Downloaded 13 Feb 2007 to 129.93.16.206. Redistribution subject
Co: $E_{F}=3.5 \mathrm{eV}, h_{e x}=1 \mathrm{eV} .{ }^{15}$ As is seen from Fig. 1(b), the conductance is different for up- and down-spin electrons and changes in discrete steps of $e^{2} / h$ with the increasing radius of the nanowire. At small values of $R$ the down-spin channel is closed, and the conductance is equal to the spin conductance quantum $e^{2} / h$. The closure of the spin channel occurs at the critical radius $R_{0}$ given by the following expression:

$$
R_{0}^{2}=\frac{2 \sqrt{m h_{e x}}}{\pi^{2} \hbar n}
$$

where $n$ is the total number of valence electrons per unit volume. In our case $R_{0} \approx 1.5 \AA$, so that the diameter of the nanowire is of the order of the lattice parameter in the bulk ferromagnet. The up-spin channel is never closed due to the charge neutrality condition imposed on the system.

In the case of a segmented nanowire with radius $R_{2}$ $<R_{1}=R_{3}$ [Fig. 1(a)], the conductance displays irregular oscillations with increasing $R_{2}$ [see Fig. 1(c)]. These oscillations are the consequence of scattering at the interfaces between the segments. Abrupt changes in the radius of the segmented wire violate the adiabatic principle ${ }^{6}$ smearing out the conductance steps. These fluctuations do not, however, prevent the closure of the down-spin channel at $R_{2}=R_{0}$, similar to the case of a wire of constant radius. At $R_{2}<R_{0}$ only the up-spin channel is open, and the electric current in the nanowire is $100 \%$ spin-polarized. We note that the vanishing up-spin conductance seen in Fig. 1(c) at small values of $R_{2}$ is the result of the potential well created within the constriction region. The charge neutrality condition requires the depth of the potential well being inversely proportional to $R_{2}^{2}$ which leads to a strong reflection of incident electronic waves at small $R_{2}$.

The possibility to achieve a fully spin-polarized conductance in the regime when only one spin-channel is open leads to another phenomenon that might occur in atomic-size contacts. If there is a nonmagnetic region within the constriction that separates two ferromagnetic electrodes, as is shown in Fig. 2(a), electronic conduction can be blocked by the spin conservation rule. Indeed, if magnetizations of the two ferromagnets are antiparallel the spin channel that is open in the one ferromagnet is closed in the other ferromagnet and vice versa. This spin blockade effect makes the conductance between the antiparallel-aligned electrodes equal to zero. This is opposite to the case of the parallel-aligned electrodes for which a conduction channel is open for up-spin electrons and the conductance is not equal to zero. We see that the magnetoresistance of such an atomic-size constriction can be infinite.

We model this effect by considering a segmented nanowire in which ferromagnetic electrodes are separated by a thin nonmagnetic spacer layer which is placed within the constriction region and has the same radius as the radius of the inner part of the nanowire, i.e., $R_{2}$ [Fig. 2(a)]. The only role of the spacer is to decouple magnetic moments of the electrodes which allows one to magnetize the ferromagnets in opposite directions. In our calculations we assume that the spacer is metallic, although the spin blockade effect survives in the case of an insulating barrier as well.

Figure 2(b) shows the conductance for parallel and antiparallel magnetization of the ferromagnets in a nanowire of AIP license or copyright, see http://apl.aip.org/apl/copyright.jsp 


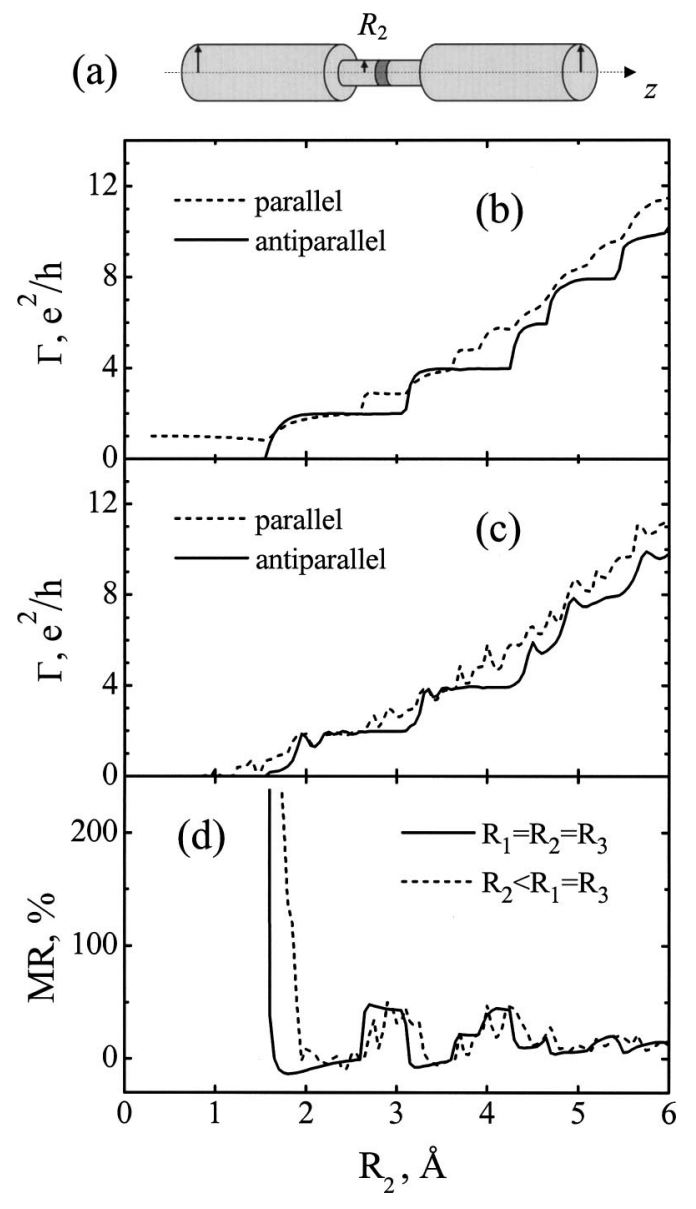

FIG. 2. (a) Segmented nanowire representing a nanoconstriction with a nonmagnetic region; (b) conductance for parallel and antiparallel alignment in a nanowire of constant radius $R=R_{1}=R_{2}=R_{3}$ as a function of $R$; (c) conductance for parallel and antiparallel alignment in a segmented nanowire of radius $R_{1}=R_{3}=15 \AA$ and $L_{2}=20 \AA$ as a function of $R_{2}$. The length of the middle segment is $2 \AA$; (d) magnetoresistance as a function of $R_{2}$.

constant radius $R=R_{1}=R_{2}=R_{3}$ as a function of $R$. As is evident from this figure, at small $R$ such that $R<R_{0}$ the spin blockade quenches the conductance of the aniparallelaligned nanowire, whereas the conductance for the parallelaligned nanowire remains nonzero, about $e^{2} / h$. This effect is also present in a segmented nanowire of radius $R_{2}<R_{1}$ $=R_{3}$ [Fig. 2(c)]. When $R_{2}$ becomes smaller than the critical radius $R_{0}$, the conductance for the antiparallel magnetization vanishes. ${ }^{16}$ Sizable fluctuations in the conductance seen in Fig. 2(c) are caused by scattering at the interfaces between the segments of different radius which irregularly changes the conductance, reflecting some specific interrelations between longitudinal momenta.

Figure 2(d) shows magnetoresistance (MR) defined by the standard ratio $\mathrm{MR}=\left(\Gamma_{P}-\Gamma_{A P}\right) / \Gamma_{A P}$, where $\Gamma_{P}$ and $\Gamma_{A P}$ are the conductance for the parallel and antiparallel magnetization of the electrodes. For the nanowire of variable radius the MR displays noisy features, but the overall dependence on $R_{2}$ is similar to that for the nanowire of constant radius. As is seen from Fig. 2(d), the MR increases at small radius of the constriction. This is consistent with the experimental observations of MR in ballistic Ni break junctions, ${ }^{17}$ which were explained in terms of a constrained domain wall formed within the nanocontact. ${ }^{15,18,19}$ At $R_{2}<R_{0}$ the MR becomes infinitely large. In this regime the nanoconstriction works as a perfect spin valve that can be switched between conducting and nonconducting states. We note, however, that this mechanism cannot explain huge values of MR in electrodeposited nanocontacts ${ }^{20}$ which were observed not only for atomic-size contacts but also for larger cross sections of the constrictions up to a few $\mathrm{nm}^{2}$

In conclusion, we have shown that atomic-size constrictions can be used to obtain a fully spin-polarized current as well as a valve which can be switched between conducting and nonconducting states. The latter can be achieved due to the spin blockade effect which quenches the conductance of the aniparallel-aligned nanowire in the regime when only one spin channel is open in each electrode.

This work is supported by NSF (Grant Nos. DMR0203359 and MRSEC: DMR-0213808), the Nebraska Research Initiative, and W. M. Keck Foundation. A.V.V. is grateful to J. Fourier University, Grenoble for hospitality and the Russian Fund for Basic Research for partial financial support.

${ }^{1}$ B. J. van Wees, H. van Houten, C. W. J. Beenakker, J. G. Williams, L. P. Kouwenhowen, D. van der Marel, and C. T. Foxon, Phys. Rev. Lett. 60, 848 (1988).

${ }^{2}$ J. I. Pascual, J. Méndez, J. Gómez-Herrero, A. M. Baró, N. García, and V. T. Binh, Phys. Rev. Lett. 71, 1852 (1993).

${ }^{3}$ L. Olesen, E. Lægsgaard, I. Stensgaad, F. Besenbacher, J. Schiøtz, P. Stoltze, K. W. Jacobsen, and J. K. Nørskov, Phys. Rev. Lett. 72, 2251 (1994).

${ }^{4}$ J. M. Krans, J. M. van Ruitenbeek, V. V. Fisun, I. K. Yanson, and L. J. de Jongh, Nature (London) 375, 767 (1995).

${ }^{5}$ R. Landauer, IBM J. Res. Dev. 32, 306 (1988).

${ }^{6}$ L. I. Glazman, G. B. Lesovik, D. E. Khmelnitskii, and R. I. Shekhter, JETP Lett. 48, 238 (1988).

${ }^{7}$ T. Ono, Y. Ooka, and H. Miyajima, Appl. Phys. Lett. 75, 1622 (1999).

${ }^{8}$ F. Elhoussine, S. Mátéfi-Tempfli, A. Encinas, and L. Piraux, Appl. Phys. Lett. 81, 1681 (2002).

${ }^{9}$ F. Komori and K. Nakatsuji, Mater. Sci. Eng., B 84, 102 (2001).

${ }^{10}$ C.-S. Yang, J. Thiltges, B. Doudin, and M. Johnson, J. Phys.: Condens. Matter 14, L765 (2002).

${ }^{11}$ W. E. Picket and J. S. Moodera, Phys. Today 5, 39 (2001).

${ }^{12}$ M. Ye. Zhuravlev, H. O. Lutz, and A. V. Vedyayev, Phys. Rev. B 63, 174409 (2001).

${ }^{13}$ A. Weisshaar, J. Lary, S. M. Goodnick, and V. K. Tripathi, J. Appl. Phys. 70, 355 (1991); A. L. Sakhnovich, J. Math. Anal. Appl. 247, 410 (2000).

${ }^{14}$ F. Kassubek, C. A. Stafford, and H. Grabert, Phys. Rev. B 59, 7560 (1999).

${ }^{15}$ H. Imamura, N. Kobayashi, S. Takahashi, and S. Maekawa, Phys. Rev. Lett. 84, 1003 (2000).

${ }^{16}$ Note that this conclusion is valid provided that no spin-flip scattering due to, e.g., spin-orbit interaction is taken into account. The spin-orbit interaction, $H_{S O}$, makes $\Gamma_{A P}$ nonzero for $R<R_{0}$. In this case the MR ratio, $\left(\Gamma_{P}-\Gamma_{A P}\right) / \Gamma_{A P}$, can be estimated as $\left(E_{F} / H_{S O}\right)^{2}$. Assuming that $H_{S O}$ $\sim 0.1 \mathrm{eV}$, we find $\mathrm{MR} \sim 10^{3}$.

${ }^{17}$ N. García, M. Muñoz, and Y.-W. Zhao, Phys. Rev. Lett. 82, 2923 (1999).

${ }^{18}$ A. K. Zvezdin and A. F. Popkov, JETP Lett. 71, 209 (2000).

${ }^{19}$ L. R. Tagirov, B. P. Vodopyanov, and K. B. Efetov, Phys. Rev. B 65, 214419 (2002).

${ }^{20}$ H. D. Chopra and S. Z. Hua, Phys. Rev. B 66, 020403 (2002). 